\title{
Ciencias Químicas
}

Citación: Galarza E. Estudios electrónicos y electroquímicos del 2,2-bis[5'- (2'-carbonilfuranil)]propano. Rev. Acad. Colomb. Cienc. Ex. Fis. Nat. 45(177):1224-1231, octubre-diciembre de 2021. doi: https://doi.org/10.18257/ raccefyn. 1401

Editor: Maria Ligia Sierra Garcia

\section{Correspondencia:}

Esperanza Galarza;

esperanza.galarza@correounivalle.edu.co

Recibido: 25 de febrero de 2021

Aceptado: 27 de agosto de 2021

Publicado: 15 de diciembre de 2021

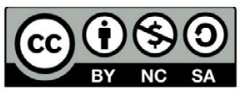

Este artículo está bajo una licencia de Creative Commons ReconocimientoNoComercial-Compartir Igual 4.0 Internacional

Artículo original

\section{Estudios electrónicos y electroquímicos del 2,2-bis [5'- (2'-carbonilfuranil)]propano}

\section{Electronic and electrochemical studies of 2,2-bis [5'- (2'-carbonylfuranyl)] propane}

\author{
(1) Esperanza Galarza \\ Grupo de investigación GISIOMCA, Departamento de Química, Universidad del Valle, Cali, Colombia
}

\section{Resumen}

En este estudio se reporta la síntesis y caracterización del compuesto 2,2-bis[5'-(2'-carbonilfuranil)] propano (BCFP), el cual es un prototipo de bloque de construcción para la preparación de macrociclos, polímeros e, incluso, estructuras supramoleculares con diferentes aplicaciones. La síntesis del BCFP se llevó a cabo a través de la reacción de Vilsmeier-Haack del 2,2-bis( 2 '-furanil) propano usando $N, N$-dimetilformamida (DMF) y cloruro de fosforilo $\left(\mathrm{POCl}_{3}\right)$, con un $55 \%$ de rendimiento. El compuesto exhibió una transición $n \rightarrow \pi^{*}$ característica de compuestos con átomos sp ${ }^{2}$ que contienen pares libres no enlazantes. Mediante voltamperometría cíclica se evidenciaron dos procesos redox (reducción y oxidación) de naturaleza electroquímica y química irreversible. Los cálculos teóricos basados en la teoría del funcional de la densidad (density functional theory, DFT) corroboraron las transiciones electrónicas y los procesos redox. Los orbitales moleculares de frontera (HOMO y LUMO) fueron dominados por los grupos aldehído y los anillos de furano.

Palabras clave: Química supramolecular;Voltamperometría cíclica; Ligandos simétricos.

\section{Abstract}

The present study reports the synthesis and characterization of the compound 2,2-bis[5'$\left(2^{\prime}\right.$-carbonylfuranyl)]propane (BCFP) which is a prototype of a building block for the preparation of macrocycles, polymers, and even supramolecular structures for different applications. The synthesis of BCFP was carried out through the Vilsmeier-Haack reaction of 2,2-bis(2'-furanyl) propane using DMF and $\mathrm{POCl}_{3}$ with a 55\% yield. The compound exhibits an $\mathrm{n} \rightarrow \pi^{*}$ transition characteristic of compounds with $\mathrm{sp}^{2}$ atoms containing free nonbonding pairs. Likewise, cyclic voltammetry (CV) showed two irreversible redox processes (reduction and oxidation) which proved to be both electrochemically and chemically irreversible in nature. Theoretical calculations at the DFT level corroborated both electronic transitions and redox processes. The frontier molecular orbitals (HOMO and LUMO) were dominated by aldehyde groups and furan rings.

Keywords: Supramolecular chemistry; Cyclic voltammetry; Symmetric ligands.

\section{Introducción}

Desde el primer reporte de su síntesis presentado por Heinrich Limpricht (Limpricht, 1870), el furano ha sido un importante punto de partida para la síntesis de una gran cantidad de derivados industriales (Hoydonckx, et al., 2007; Fauque, 1954) y moléculas de interés en química orgánica (Katritzky, et al., 2010).

Los derivados de furano se conocen ampliamente como bloques de construcción para la preparación de polímeros (Gaitonde, et al., 2014; Gandini \& Belgacem, 1997; Moore, et al., 1978; Moore \& Kelly, 1978; Moore \& Kelly, 1979; Moore \& Kelly, 1984, Zeng, et al., 2013) y su impacto en el desarrollo tecnológico agrícola y farmacéutico, así como en procesos sintéticos, se reconoce desde hace décadas (Peters, 1939). 
Dada su gran importancia sintética y aplicativa, se emprendió la síntesis de un derivado de furano, específicamente el 2,2-bis[5'-(2'-carbonilfuranil)]propano (BCFP), utilizando la reacción de Vilsmeier-Haack. Este compuesto se presenta como un prototipo de molécula que podría emplearse como monómero en reacciones de polimerización $\mathrm{y}$, gracias a su simetría, también como bloque de construcción en las estructuras metal-orgánicas (metalorganic frameworks, MOFs).

En el presente estudio se reporta la síntesis y caracterización del compuesto BCFP, cuyas propiedades electrónicas se estudiaron por espectroscopia UV-Vis, en tanto que las electroquímicas se analizaron por voltamperometría cíclica. Los resultados obtenidos se contrastaron con cálculos teóricos hechos con el programa Gaussian 16 usando el funcional B3LYP y una base 6-31+G (d,p). El objetivo fue resaltar la importancia de este compuesto como punto de partida en química supramolecular y explorar por primera vez las propiedades electrónicas y electroquímicas que permitan potenciar su utilidad.

\section{Materiales y métodos}

El seguimiento de las reacciones y la verificación de la pureza de los productos se hizo por cromatografía de capa delgada (CCD) empleando placas de gel de sílice 60F 254 con un espesor de 0,2 mm (Merck); como revelador se utilizó una lámpara Spectroline serie E con dos longitudes de onda, 254 y $363 \mathrm{~nm}$. Los reactivos y solventes se adquirieron de las casas comerciales Sigma-Aldrich ${ }^{\circledR}$ y Merck ${ }^{\circledR}$ y se utilizaron sin purificación adicional. Los solventes eran de grado analítico y algunos se secaron según los protocolos establecidos.

Los puntos de fusión se determinaron con un fusiómetro Stuart SMP3 o Barnstead Electrothermal modelo IA9300. Los espectros de infrarrojo (IR) se tomaron en un equipo FT-IR 8400 equipado con accesorio de ATR con punta de diamante. En un espectrómetro Bruker UltrashieldTM de $400 \mathrm{MHz}$, operado a $400 \mathrm{MHz}$ para ${ }^{1} \mathrm{H}$ y $100 \mathrm{MHz}$ para ${ }^{13} \mathrm{C}$, se capturaron los espectros de RMN utilizando como solvente $\mathrm{CDCl}_{3}-d$. Además, se hicieron los experimentos bidimensionales HSQC, DEPT-135, HMBC, COSY y NOESY para verificar la naturaleza del producto.

La composición porcentual de los elementos de carbono e hidrógeno se determinó en un analizador elemental Thermo Flash EA 1112 Series y las mediciones de absorbancia se hicieron con un espectrofotómetro Pharma Spec Shimadzu JASCO V-730 UV-VIS. Los cálculos computacionales se hicieron con el programa Gaussian 16 usando el funcional B3LYP y una base $6-31+\mathrm{G}(\mathrm{d}, \mathrm{p})$. Las imágenes de orbitales moleculares de frontera se obtuvieron con la aplicación Gauss View 6.

Las pruebas de electroquímica se realizaron en un galvanostato potenciostato Metrohm Autolab PGSTAT302N utilizando como electrodo de trabajo carbón vítreo de $3 \mathrm{~mm}$ de diámetro, un alambre de platino como electrodo de conteo y un alambre de plata como electrodo de pseudorreferencia. Se agregó ferroceno sublimado como estándar interno para reportar los picos redox. Antes de iniciar las mediciones se dejó pasar un flujo de nitrógeno o argón (a través de la solución) para eliminar el oxígeno del medio. El comportamiento electroquímico se analizó mediante voltamperometría cíclica y de onda cuadrada (OSWV) empleando DMF previamente secada y hexafluorofosfato de tetrabutilamonio $(n-\mathrm{Bu})_{4} \mathrm{NPF}_{6}$ como electrólito de soporte $(0,1 \mathrm{M})$. Cada experimento se realizó a diferentes velocidades de barrido a partir de los $50 \mathrm{mV} / \mathrm{s}$ y hasta los $500 \mathrm{mV} / \mathrm{s}$.

\section{2,2-bis[5'-(2'-carbonilfuranil)]propano (BCFP)}

Para la síntesis de este compuesto se siguió el protocolo reportado en la literatura (Chen \& $\mathbf{W u}, 2002)$. Se adicionó $\mathrm{POCl}_{3}$ (1,2 equivalentes) gota a gota a una solución que contenía un equivalente de 2,2-di(2-furil)propano (TCI Chemicals) en $5 \mathrm{~mL}$ de DMF bajo atmósfera de nitrógeno en constante agitación y sobre un baño de hielo. Después de mezclados, se agitó la solución por 5 horas en un baño de agua a $50{ }^{\circ} \mathrm{C}$ hasta que la solución se tornó color vino tinto, punto en el cual se dejó enfriar a temperatura ambiente. Posteriormente, se adicionaron gota a gota $10 \mathrm{~mL}$ de DMF y un (1) equivalente de $\mathrm{POCl}_{3}$ a la solución en un baño de hielo. La reacción continuó durante 10 horas en un baño de agua a $50{ }^{\circ} \mathrm{C}$ que luego 
se enfrió a temperatura ambiente y finalmente se vertió en agua-hielo. La mezcla resultante se neutralizó con hidróxido de sodio $(\mathrm{NaOH})$ al $10 \%$ y se dejó en reposo. Transcurridas 12 horas, se observó un sólido amarillo pálido que fue filtrado y recristalizado dos veces en etanol, resultando en un sólido blanco con un rendimiento del $55 \%$. RMN ${ }^{1} \mathrm{H}\left(\mathrm{CDCl}_{3}, 400\right.$ $\mathrm{MHz}) \delta(\mathrm{ppm}): 9.63$ (s, 2H, CHO), 7.22 (d, J=3.1 Hz, 2H, ArH), 6.37 (d, J=3.2 Hz, 2H, Ar), $1.79(\mathrm{~s}, 6 \mathrm{H}, \mathrm{CH} 3) . \mathrm{RMN}{ }^{13} \mathrm{C}\left(\mathrm{CDCl}_{3}, 100 \mathrm{MHz}\right) \delta(\mathrm{ppm}): 26,38,108,123,152,164,178$. IR $(\mathrm{KBr}) v\left(\mathrm{~cm}^{-1}\right):$ 1684, $1669(\mathrm{C}=\mathrm{O}), 1374,1361(\mathrm{C}-\mathrm{H}), 1241,968(\mathrm{C}-\mathrm{O}-\mathrm{C}) . \mathrm{MS}(\mathrm{EI}) \mathrm{m} / \mathrm{z}$ 232,217 (M-15). Punto de fusión: $91-93^{\circ} \mathrm{C}$. Análisis elemental calculado para $\mathrm{C}_{13} \mathrm{H}_{12} \mathrm{O}_{4}: \mathrm{C}$ $67,23 \%$, H 5,21 \%, O 27,56 \%. Experimental C 67,22 \%, H 5,21 \%, O 27,57 \%.

\section{Resultados y discusión}

La reacción de Vilsmeier-Haack es una reacción de una amida sustituida con tricloruro de fosforilo y un areno activado. La reacción fue publicada por primera vez en 1927 por Anton Vilsmeier y Albrecht Haack (Vilsmeier \& Haack, 1927) y desde entonces se ha utilizado ampliamente en la síntesis de aldehídos.

El BCFP se preparó a partir del compuesto comercial 2,2-di(2-furil)propano, el cual actúa como arilo activado (Figura 1). En el presente caso, la DMF fue una amina sustituida que reaccionó con el tricloruro de fosforilo para formar el ion cloroiminio (reactivo de Vilsmeier), el cual reaccionó a su vez con el 2,2- di(2-furil)propano (arilo activado) a través de una reacción de sustitución aromática electrofílica generando un ion iminio intermedio que luego se hidrolizó para obtener el compuesto objetivo. La reacción requirió ajustar el $\mathrm{pH}$ con $\mathrm{NaOH}$ (al $10 \%$ ) dado el aumento de acidez ocasionado por la presencia del subproducto protonado de la dimetilamina. Para garantizar que ambos anillos fueran formilados fue necesario prolongar la reacción y realizar dos adiciones de DMF y cloruro de fosforilo. El rendimiento de la reacción resultó aceptable (55\%), aunque ligeramente menor al reportado previamente (Chen $\& \mathbf{W u}, 2002)$. Cabe destacar que, a pesar de que la reacción de Vilsmeier-Haack es ampliamente conocida, en el caso de la síntesis del BCFP se requirieron múltiples ensayos debido al poco rendimiento producto de la no formilación paralela de los anillos. Al final del protocolo empleado se introdujo una modificación al reportado en la literatura (Chen $\& \mathbf{W u}, 2002)$, con la cual se obtuvo el mejor rendimiento.

Los espectros de RMN ( ${ }^{1} \mathrm{H} \mathrm{y}{ }^{13} \mathrm{C}$ ) (Figura 2) mostraron las señales esperadas para el compuesto. Debido a su simetría, se observaron cuatro señales en el RMN protónico: la primera, un singulete que integra para dos protones, se localizó en las 9,63 ppm, lo que es típico de los aldehídos. La segunda señal, un doblete que apareció en las 7,22 ppm, integra para dos protones con una constante de acoplamiento J de $3.1 \mathrm{~Hz}$ y correspondió al protón 4 del anillo furano (marcado con un punto verde). Una tercera señal se observó en $6,37 \mathrm{ppm}$, que integra para dos protones con una constante de acoplamiento $\mathrm{J}$ de $3.2 \mathrm{~Hz}$, y correspondió al protón 3 del anillo furano (señalado en rojo en la figura). Por último, en el campo de arriba se observó un singulete que integra para seis protones, el cual se asignó a los protones metílicos. Asimismo, el RMN ${ }^{13} \mathrm{C}$ (Figura 2) evidenció las 7 señales esperadas del compuesto BCFP. La asignación de dichas señales, así como las de $\mathrm{RMN}{ }^{1} \mathrm{H}$, se basaron en los resultados bidimensionales (COSY, HMBC, DEPT 135) de la RMN.

El espectro UV-Vis se tomó en cloroformo (Figura 3). Se observó una absorción principal en los $293 \mathrm{~nm}$, con un hombro en los $276 \mathrm{~nm}$. Dichas absorciones se desplazaron hipsocrómicamente al aumentar la polaridad del solvente (metanol), efecto debido

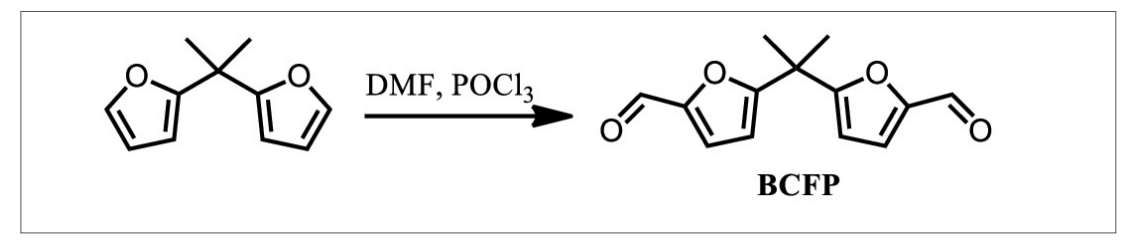

Figura 1. Síntesis del 2,2-bis[5'-(2'-carbonilfuranil)]propano a partir de DMF y $\mathrm{POCl} 3$ 
al incremento en la solvatación del par electrónico libre de los oxígenos de las unidades furano (el otro par hace parte de la resonancia del anillo), lo cual tiene como consecuencia la disminución de la energía del orbital $n$. La transición de un electrón al orbital $\pi^{*}$ produjo un estado excitado, cuyo nivel de energía no se vio afectado por el enlace de hidrógeno entre el metanol o los oxígenos. De esta forma, la energía asociada a la transición $n \rightarrow \pi^{*}$ se incrementó (reducción en la energía del orbital n). Por su parte, el hombro observado a 276 $\mathrm{nm}$ se asoció con la absorción de los grupos formilo laterales y exhibió el mismo fenómeno.
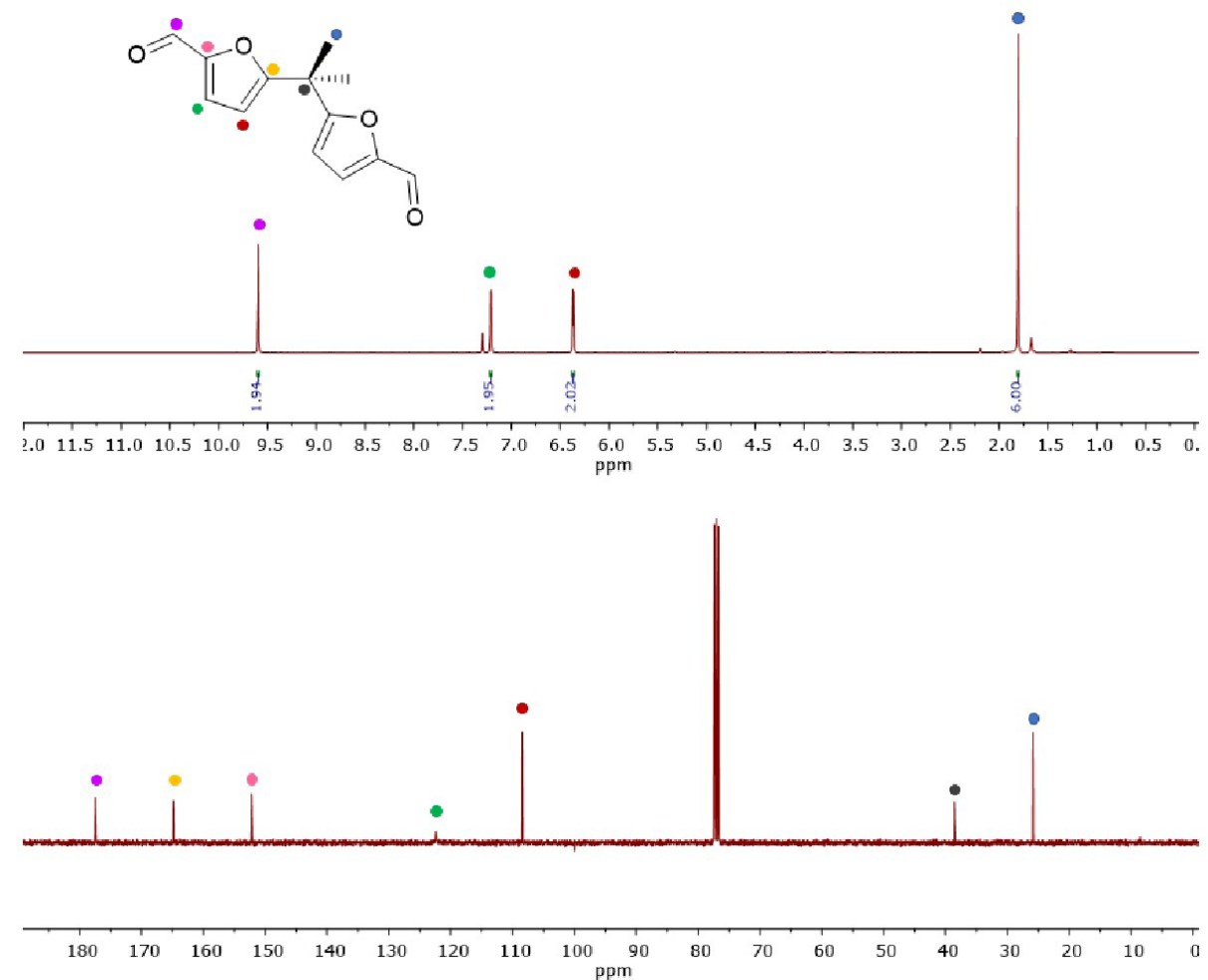

Figura 2. (Arriba) Espectro de $\mathrm{RMN} 1 \mathrm{H}(\mathrm{CDCl} 3,400 \mathrm{MHz})$. (Abajo) Espectro de RMN 13C (CDCl3, $100 \mathrm{MHz})$

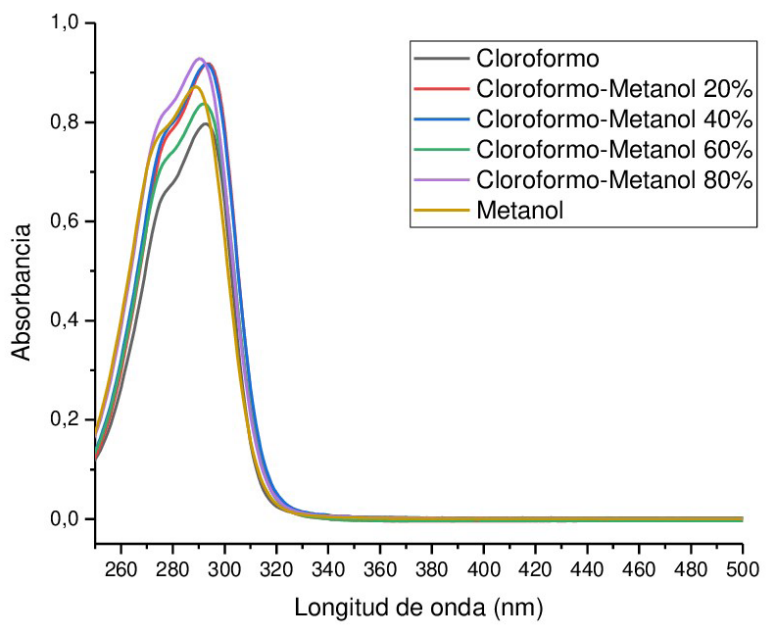

Figura 3. Espectro de absorción UV-Vis del compuesto BCFP en cloroformo y gradientes del solvente cloroformo-metanol 
Se optimizó, asimismo, la geometría de la molécula BCFP utilizando el programa Gaussian 16 (Frisch, et al., 2016) con el funcional B3LYP y la base 6-31+G (d,p). Como estructura de entrada se utilizaron los datos cristalográficos reportados por Gaitonde, et al. (2014) para una molécula similar. Los cálculos sirvieron para estimar las energías de los orbitales moleculares de frontera HOMO y LUMO (Figura 4), con el fin de correlacionar sus superficies con los resultados obtenidos por medidas electroquímicas y de UV-Vis.

La unidad básica del compuesto BCFP es el furfural, el cual tiene múltiples aplicaciones a nivel industrial y de química fina (Eseyinc \& Steele, 2015). Sus propiedades electroquímicas ya se conocen y muchas de sus aplicaciones se centran en la oxidación a ácido furoico u otros ácidos derivados. Asimismo, la reducción del furfural permite la obtención del alcohol furfuril (Mariscal, et al., 2016; da Silva, et al., 2017). En este sentido, se esperaba un comportamiento similar en el compuesto analizado en el presente estudio, con orbitales moleculares de frontera de una densidad electrónica localizada principalmente sobre los anillos de furano y los aldehídos. Los grupos metílicos actuaron como donantes de densidad electrónica, de ahí su baja contribución al orbital LUMO. Los anillos furano en el compuesto BCFP poseen seis electrones $\pi$ deslocalizados entre los cinco átomos que conforman el anillo, siendo un compuesto aromático $\pi$ excedente, por lo que se esperaba que el comportamiento electroquímico del compuesto estuviera gobernado por estas características electrónicas. Tal comportamiento también se observó en la UV-Vis, en la que la principal transición electrónica se debe al anillo furano con un hombro producto de transiciones en los grupos carbonilo de los aldehídos. La voltamperometría cíclica ha sido una técnica electroquímica ampliamente utilizada para entender los mecanismos y propiedades oxidativas o reductivas frente a procesos de transferencia de carga tanto en compuestos orgánicos como inorgánicos. Los procesos catódicos y anódicos del compuesto BCFP se muestran en las Figuras 5-7.

El compuesto BCFP exhibió un potencial de oxidación a $0,87 \mathrm{~V}$, irreversible desde el punto de vista electroquímico, ya que no se observó una onda de reducción al escanear en la dirección catódica (Figura 5), lo que es indicativo de que el compuesto sufre un proceso químico que degrada o convierte la molécula en otra especie no activa dentro de la ventana de potencial de trabajo.

Cuando se aumentó la velocidad de escaneo de $50 \mathrm{mV} / \mathrm{s}$ a $500 \mathrm{mV} / \mathrm{s}$, se observó un aumento en la intensidad de corriente para el proceso de oxidación (Figura 6) debido a que el aumento de la velocidad de escaneo disminuye el grosor de la capa de difusión en la interfase electrodo-solución. También se observó un evento reductivo, de baja intensidad, alrededor de los $-0,15 \mathrm{~V}$, más notorio al aumentar la velocidad de escaneo, lo cual es indicativo de que el comportamiento electroquímico de este compuesto en potenciales

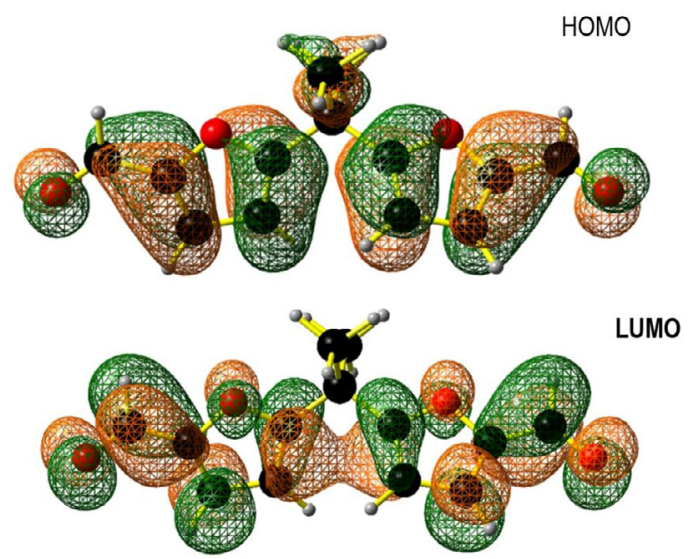

Figura 4. Representación tridimensional del HOMO (arriba) y LUMO (abajo) calculados para la molécula BCFP. Paquete gráfico: Gaussian View. Cálculos llevados a cabo con Gaussian 16 usando el funcional B3LYP y la base $6-31+G(d, p)$ 


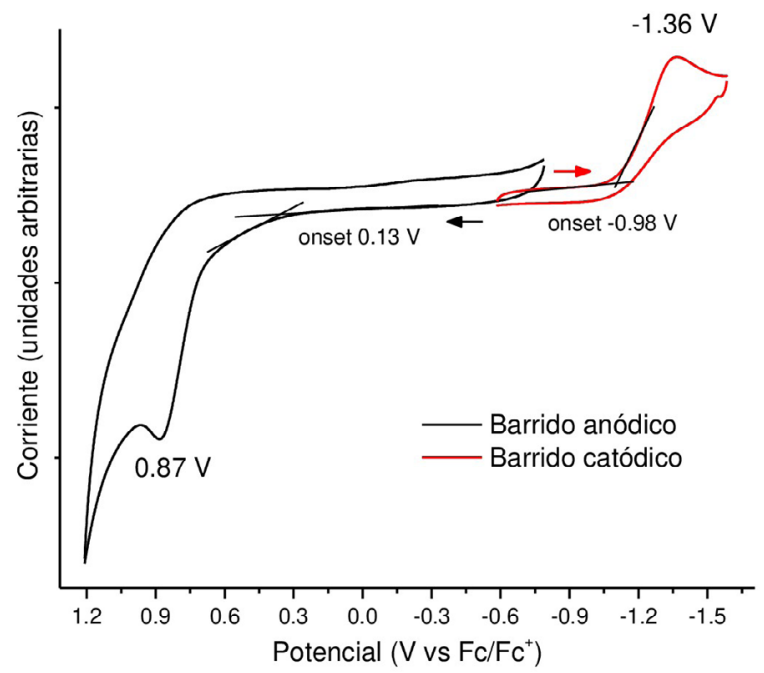

Figura 5. Voltamperograma cíclico del compuesto BCFP. Solución de $0,1 \mathrm{M}$ de (n-Bu)4NPF6 en DMF. Se usaron carbón vítreo como electrodo de trabajo, alambre de plata como pseudo-referencia y alambre de platino como electrodo de conteo. Velocidad de escaneo: $100 \mathrm{mV} / \mathrm{s}$

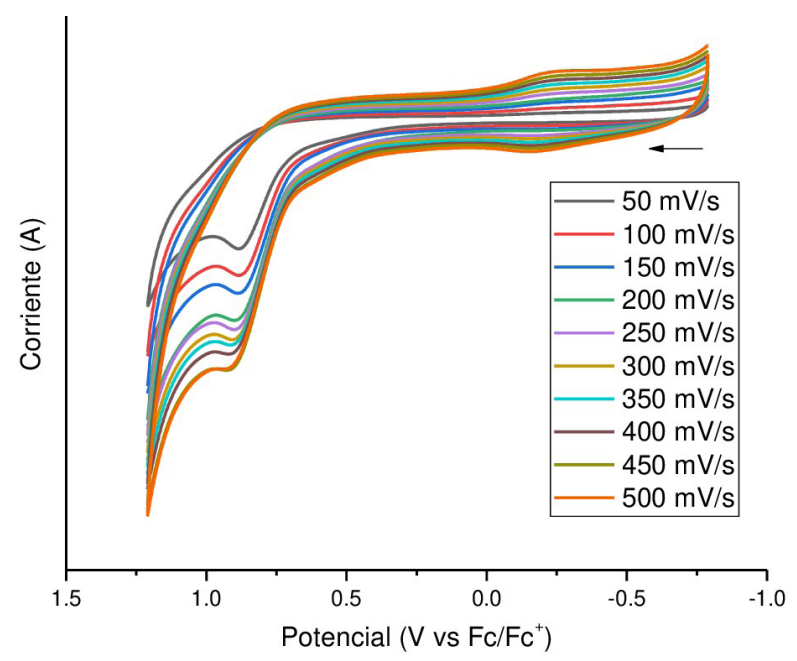

Figura 6. Voltamperograma cíclico (escaneo anódico a diferentes velocidades) del compuesto BCFP. Solución de 0,1 M de (n-Bu)4NPF6 en DMF. Se emplearon carbón vítreo como electrodo de trabajo, alambre de plata como pseudo-referencia y alambre de platino como electrodo de conteo

anódicos es una combinación de una transferencia electrónica lenta y una reacción química rápida. Por último, el análisis de la ecuación de Randles-Sevcik evidenció que la intensidad de corriente $\left(\mathrm{i}_{\mathrm{p}}\right)$ no comportaba una dependencia lineal con la raíz cuadrada de la velocidad de escaneo $\left(\mathrm{v}^{1 / 2}\right)$ y tampoco se encontró relación lineal entre $\mathrm{i}_{\mathrm{p}} \mathrm{y} \mathrm{v}$, por lo cual se presumió que el proceso de transferencia electrónica ocurrió a través de especies adsorbidas a la superficie del electrodo.

En el caso del escaneo en potenciales catódicos, el compuesto BCFP exhibió una reducción electroquímica y químicamente irreversible. El aumento en la velocidad de escaneo no reflejó una reversibilidad, por lo menos apreciable, en el rango de las velocidades de escaneo utilizadas (Figura 7), y en la oxidación tampoco se observó una dependencia lineal de la corriente de pico con la velocidad de escaneo o su raíz cuadrada ni la presencia de pre-picos que pudieran sugerir procesos de adsorción evidentes. 


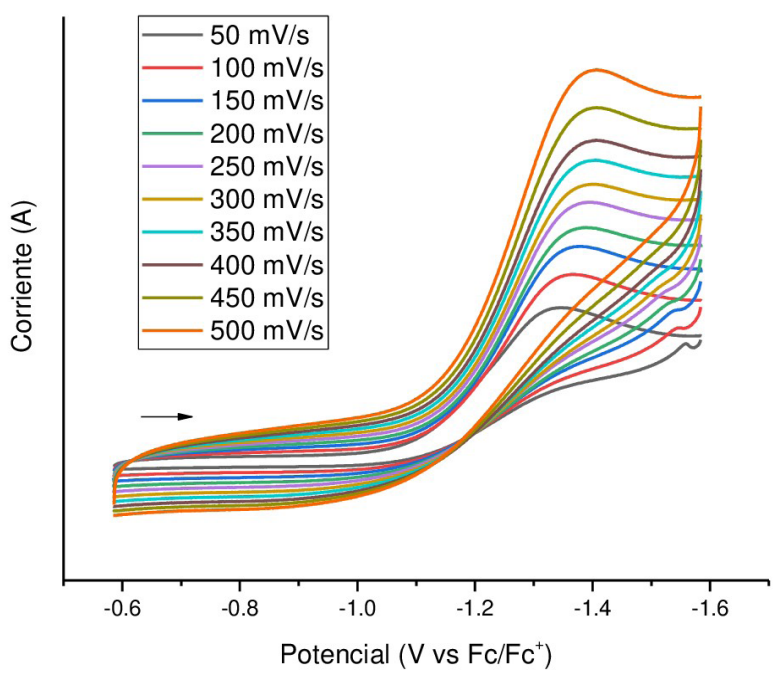

Figura 7. Voltamperograma cíclico (escaneo catódico a diferentes velocidades) del compuesto BCFP. Solución de 0,1 M de (n-Bu)4NPF6 en DMF. Se emplearon carbón vítreo como electrodo de trabajo, alambre de plata como pseudo-referencia y alambre de platino como electrodo de conteo

$\mathrm{Al}$ analizar la información electroquímica del compuesto BCFP, se observó que la intensidad de la onda de reducción fue mucho mayor que la del pico de oxidación, lo que lleva a pensar que el número de electrones involucrados en cada proceso es diferente y que, al comparar la intensidad de los picos y sus áreas, podrían representarse como dos procesos redox con diferente número de electrones involucrados: el primero, una oxidación en la que se abstrae un electrón para generar un catión radical, y la segunda, una reducción que involucra la transferencia de dos electrones (Figura 1S, https://www.raccefyn.co/ index.php/raccefyn/article/view/1401/3133). En ambos casos las especies resultantes reaccionaron químicamente de manera rápida y de ahí la irreversibilidad observada.

Gracias a la correlación entre los cálculos DFT y los voltamperogramas, en los que se observó en el LUMO una mayor localización en los carbonilos, en tanto que en los HOMO la localización estuvo sobre los anillos (Figura 4), se propone que la reducción ocurre sobre los aldehídos, lo que posiblemente conlleva a que estos pasen a formar alcoholes si hay trazas de agua o acidez en el medio. En el caso de la oxidación, esta se llevaría a cabo principalmente sobre el anillo furano, generando un radical catión con subproductos no activos electroquímicamente en la ventana de potencial en que se trabajó.

Los resultados obtenidos muestran que la molécula BCFP es termodinámicamente estable $(\Delta \mathrm{E}=2,23 \mathrm{~V})$, con transiciones electrónicas principalmente en la región ultravioleta y una química regida por los grupos aldehído y la $\pi$-excedencia de los anillos aromáticos. Estas características lo sitúan como un buen candidato a bloque de construcción de arquitecturas supramoleculares. Por ejemplo, la reacción del BCFP con dos equivalentes de la 2-hidracinopiridina resultaría en un ligando ditópico tridentado, asemejando en cada extremo el bolsillo de una terpiridina. Este tipo de ligandos pueden aprovecharse para formar rejillas moleculares al coordinar iones metálicos (de coordinación octaédrica o tetraédrica).

\section{Conclusiones}

Se preparó un compuesto dialdehído simétrico (BCFP) basado en dos anillos de furano interconectados por un grupo metileno. La metodología de síntesis se basó en la reacción de Vilsmeier-Haack. El compuesto se caracterizó mediante resonancia magnética nuclear $\left({ }^{1} \mathrm{H} \mathrm{y}{ }^{13} \mathrm{C}\right)$, FT-IR, espectrometría de masas, análisis elemental, espectroscopía UV-Vis, voltamperometría cíclica y cálculos computacionales a nivel de DFT. Los procesos electro-químicos que sufrió el compuesto fueron irreversibles al igual que las 
reacciones químicas que siguieron. Los datos de corriente de pico versus velocidad de escaneo sugieren que los procesos de transferencia de carga son mediados a través de especies adsorbidas.

\section{Agradecimientos}

Al profesor Manuel N. Chaur del departamento de Química de la Universidad del Valle, por su aporte a las discusiones sobre el estudio electroquímico del compuesto y otros en perspectiva.

\section{Información suplementaria}

Figura 1S. Procesos redox observados para el BCFP. Ver figura 1S en https://www.raccefyn. co/index.php/raccefyn/article/view/1401/3133

\section{Conflicto de intereses}

La autora declara no tener ningún conflicto de intereses con respecto a este estudio.

\section{Referencias}

Chen, Z.-H. \& Wu, C.-T. (2002). Synthesis of Schiff's base macrocyclic compounds containing furan ring. Chinese Journal of Organic Chemistry. 22 (8): 582-586.

da Silva, J. L., Beloumini, M. A., Stradiotto, N. R. (2017). Cathodic electrochemical determination of furfural in sugarcane bagasse using an electrode modified with nickel nanoparticles. Analytical Methods. 9 (5): 826-834.

Eseyin, A. E. \& Steele, P. H. (2015). An overview of the applications of furfural and its derivatives. International Journal of Advanced Chemistry. 3 (2): 42-47.

Fauque, L. L. J. (1954). U.S. Patent 2,681,917.

Frisch, N. M. J., Trucks, G. W., Schlegel, H. B., Scuseria, G. E., Robb, M. A., Cheeseman, J. R., Fox, D. J. (2016). Gaussian 16, Revision B.01, Gaussian, Inc., Wallingford CT.

Gaitonde, V., Lee, K., Kirschbaum, K., Sucheck, S. J. (2014). Bio-based bisfuran: synthesis, crystal structure, and low molecular weight amorphous polyester. Tetrahedron Letters. 55: 4141-4145.

Gandini, A. \& Belgacem, M. N. (1997). Furans in polymer chemistry. Progress in Polymer Science. 22 (7): 1203-1379.

Hoydonckx, H. E., Van Rhijn, W. M., Van Rhijn, W., De Vos, D. E., Jacobs, P. A. (2007). Furfural and Derivatives. En Ullmann's Encyclopedia of Industrial Chemistry. Weinheim, Alemania: Wiley-VCH. Doi: 10.1002/14356007.a12_119.pub2

Katritzky, A.R., Ramsden, C.A., Joule, J.A., Zhdankin, V.V. 3.3 - Reactivity of Five-Membered Rings with One Heteroatom, Editor(s): Alan R. Katritzky, Christopher A. Ramsden, John A. Joule, Viktor V. Zhdankin. Handbook of Heterocyclic Chemistry (Third Edition), Elsevier, 2010, p. 383-472, ISBN 9780080958439. Doi: 10.1016/B978-0-08-095843-9.00009-4

Limpricht, H. (1870). Ueber das Tetraphenol $\mathrm{C}_{4} \mathrm{H}_{4} \mathrm{O}$. Berichte der Deutschen Chemischen Gesellschaft. 3 (1): 90-91.

Mariscal, R., Maireles-Torres, P., Ojeda, M., Sádaba, I., López-Granados, M. (2016). Furfural: a renewable and versatile platform molecule for the synthesis of chemicals and fuels. Energy \& Environmental Science. 9 (4): 1144-1189.

Moore, J. \& Kelly, J. E. (1978). Polyesters Derived from Furan and Tetrahydrofuran Nuclei. Macromolecules. 11 (3): 568-573.

Moore, J. \& Kelly, J. E. (1978). Thermally initiated crosslinking of an unsaturated heterocyclic polyester. Journal of Polymer Science Part A: Polymer Chemistry. 16 (9): 2407-2409.

Moore, J. \& Kelly, J. E. (1979). Polymerization of furandicarbonyl chloride with bisphenol A poly (2,5-furandiylcarbonyloxy-1,4-phenylenedimethylmethylene-1,4-phenyleneoxycarbonyl). Polymer. 20 (5): 627-628.

Moore, J. \& Kelly, J. E. (1984). Polyhydroxymethylfuroate [poly(2,5-furandiylcarbonyloxy methylene)]. Journal of Polymer Science Part A: Polymer Chemistry. 22 (3): 863-864.

Peters, F. N. Jr. (1939). Industrial Uses of Furans. Industrial and Engineer Chemistry. 31 (2): 178-180.

Vilsmeier, A. \& Haack, A. (1927). Über die Einwirkung von Halogenphosphor auf Alkyl-formanilide. Eine neue Methode zur Darstellung sekundärer und tertiärer p-Alkylamino-benzaldehyde. Berichte der Deutschen Chemischen Gesellschaft zu Berlin. 60 (1): 119-122.

Zeng, C., Seino, H., Ren, J., Hatanaka, K., Yoshie, N. (2013). Bio-Based Furan Polymers with Self-Healing Ability. Macromolecules. 46 (5): 1794-1802. 